\title{
Hermite Matrix Polynomial Collocation Method for Linear Complex Differential Equations and Some Comparisons
}

\author{
Mina Bagherpoorfard, Fahime Akhavan Ghassabzade* \\ Department of Mathematics, Islamic Azad University, Fasa Branch, Fasa, Iran \\ Email: mi.bagherpoorfard@um.ac.ir, "akhavan_gh@yahoo.com
}

Received August 20, 2013; revised September 25, 2013; accepted October 1, 2013

Copyright (C) 2013 Mina Bagherpoorfard, Fahime Akhavan Ghassabzade. This is an open access article distributed under the Creative Commons Attribution License, which permits unrestricted use, distribution, and reproduction in any medium, provided the original work is properly cited.

\begin{abstract}
In this paper, we introduce a Hermite operational matrix collocation method for solving higher-order linear complex differential equations in rectangular or elliptic domains. We show that based on a linear algebra theorem, the use of different polynomials such as Hermite, Bessel and Taylor in polynomial collocation methods for solving differential equations leads to an equal solution, and the difference in the numerical results arises from the difference in the coefficient matrix of final linear systems of equations. Some numerical examples will also be given.
\end{abstract}

Keywords: Approximate Solution; Collocation Methods; Complex Differential Equations; Hermite Polynomials; Operational Matrix

\section{Introduction}

Complex differential equations and their solutions play a major role in science and engineering. A physical event can be modeled by complex differential equations. Since a few of these equations cannot be solved explicitly, it is often necessary to resort to approximation and numerical techniques. In recent years, the studies on complex differential equations were developed very rapidly [1-6].

Since 1994, matrix polynomial collocation approaches such as Taylor and Bessel matrix collocation methods have been used by Sezer and colleagues [7-11] to solve the complex linear differential equations.

The present work contains two main parts, in the first part, we use Hermite matrix collocation method to find the approximate solution of higher-order linear complex differential equations of the following form.

$$
\sum_{k=0}^{n} p_{k}(z) f^{(k)}=g(z),
$$

which is a generalized case of the complex differential equations given in $[5,6]$, with themixed conditions

$$
\sum_{k=0}^{m-1} \sum_{j=0}^{J} a_{r k} f^{(k)}\left(\xi_{j}\right)=\lambda_{r} ; r=0,1, \cdots, m-1
$$

in the following rectangular domain

*Corresponding author.

$$
D=\{z=x+i y, z \in c, a \leq x \leq b, c \leq y \leq d, a, b, c, d \in R\},
$$

or elliptic domain

$$
\begin{aligned}
D=\{ & \{z=x+i y, z \in c, x=a \cos (\theta), y=b \sin (\theta), \\
& 0<\theta \leq 2 \pi, a \leq x \leq-a, b \leq y \leq-b, a, b \in R+\}
\end{aligned}
$$

In the second part, we will study the effect of using different polynomial classes on the matrix polynomial methods.

The outline of this paper is as follows. In Section 2, we briefly introduce Hermite polynomial and describe details of using these polynomials in matrix polynomial collocation method. Section 3 focuses on the comparison of matrix collocation methods when different polynomials are used. We present the results of numerical experiments in Section 4. Finally, conclusions are drawn in Section 5 .

\section{Hermite Matrix Polynomial Collocation Method}

In this section, we describe the matrix form of Hermite polynomials and Hermite collocation Method for complex differential equations. Our aim is to find an ap- 
proximate solution of (1) defined by a truncated Hermite series form

$$
f_{N}(z)=\sum_{n=0}^{N} a_{n} H_{n}(z)=H(z) A,
$$

where $H(z)=\left[\begin{array}{llll}H_{0}(z) & H_{1}(z) & \cdots & H_{N}(z)\end{array}\right]$ such that $H_{n}(z), n=0,1, \cdots, N$ are the Hermite polynomials defined by

$$
H_{n}(z)=n ! \sum_{j=0}^{N}(-1)^{j} \frac{2^{n-2 j}}{j !(n-2 j !)} z^{n-2 j},
$$

where $N=\frac{n}{2}$ if $n$ is even and $N=\frac{n-1}{2}$ if $n$ is odd and $A=\left[\begin{array}{llll}a_{1} & a_{2} & \cdots & a_{N}\end{array}\right]^{\mathrm{T}}$. It is well known [12] that the relation between the powers $z_{N}$ and Hermite polynomials is

$$
M=\left[\begin{array}{cccccc}
1 & 0 & 0 & 0 & \cdots & 0 \\
0 & \frac{1}{2} & 0 & 0 & \cdots & 0 \\
\frac{1}{2} & 0 & \frac{1}{4} & 0 & \cdots & 0 \\
0 & \frac{3}{4} & 0 & \frac{1}{8} & \cdots & 0 \\
\vdots & \vdots & \vdots & \vdots & \ddots & \vdots \\
0 & \frac{N !}{2^{N}\left(\frac{N-1}{2}\right) ! 1 !} & 0 & \frac{N !}{2} 2^{N}\left(\frac{N-1}{2}-1\right) ! 3 ! & & 0
\end{array}\right]
$$

and for even $N$

$$
M=\left[\begin{array}{cccccc}
1 & 0 & 0 & 0 & \cdots & 0 \\
0 & \frac{1}{2} & 0 & 0 & \cdots & 0 \\
\frac{1}{2} & 0 & \frac{1}{4} & 0 & \cdots & 0 \\
0 & \frac{3}{4} & 0 & \frac{1}{8} & \cdots & 0 \\
\vdots & \vdots & \vdots & \vdots & \ddots & \vdots \\
\frac{N !}{2^{N}\left(\frac{N}{2}\right) ! 0 !} & 0 & \frac{N !}{2^{N}\left(\frac{N}{2}-1\right) ! 2 !} & 0 & \cdots & \frac{N !}{2^{N}(0) ! N !}
\end{array}\right]
$$

Then, by taking into account (5), we obtain $H(z)=Z(z)\left(M^{-1}\right)^{\mathrm{T}}$ and we can replace series (6) in the matrix form

$$
f_{N}(z)=H(z) A=Z(z)\left(M^{-1}\right)^{\mathrm{T}} A .
$$

Furthermore, the relation between the matrix $Z(z)$ and its derivative $Z^{(1)}(z)$ is

$$
Z^{(1)}(z)=Z(z) B^{T}
$$

$$
Z^{2 n}=\frac{(2 n) !}{2^{2 n}} \sum_{n=0}^{r} \frac{H_{2 n}(z)}{(r-n) ! 2 n !^{\prime}} ; 0 \leq|z| \leq 1,
$$

and

$$
Z^{2 n+1}=\frac{(2 n+1) !}{2^{2 n+1}} \sum_{n=0}^{r} \frac{H_{2 n+1}(z)}{(r-n) !(2 n+1) !} ; 0 \leq|z| \leq 1
$$

By using the expression (6) and (7) and taking $n=0,1, \cdots, N$ we find the corresponding matrix relation as follows $Z(z)^{\mathrm{T}}=M(H(z))^{\mathrm{T}}$, and

$$
Z(z)=H(z) M^{\mathrm{T}},
$$

where $Z(z)=\left[\begin{array}{lllll}1 & Z & z^{2} & \cdots & z^{N}\end{array}\right]$, for odd $N$

where

where

$$
B^{\mathrm{T}}=\left[\begin{array}{ccccc}
0 & 1 & 0 & \cdots & 0 \\
0 & 0 & 2 & \cdots & 0 \\
\vdots & \vdots & \vdots & \ddots & \vdots \\
0 & 0 & 0 & \cdots & N \\
0 & 0 & 0 & \cdots & 0
\end{array}\right]
$$

And 


$$
\left(B^{\mathrm{T}}\right)^{0}=\left[\begin{array}{ccccc}
1 & 0 & 0 & \cdots & 0 \\
0 & 1 & 0 & \cdots & 0 \\
\vdots & \vdots & \vdots & \ddots & \vdots \\
0 & 0 & 0 & \cdots & 1
\end{array}\right]
$$

From the matrix Equation (10), we get the following relations:

$$
\begin{gathered}
Z^{(2)}(z)=Z^{(1)}(z) B^{\mathrm{T}}=Z(z)\left(B^{\mathrm{T}}\right)^{2}, \\
Z^{(3)}(z)=Z^{(2)}(z) B^{\mathrm{T}}=Z(z)\left(B^{\mathrm{T}}\right)^{3}, \\
\vdots \\
Z^{(k)}(z)=Z^{(k 1)}(z) B^{\mathrm{T}}=Z(z)\left(B^{\mathrm{T}}\right)^{k} .
\end{gathered}
$$

By using relations (9) and (11), we have a recurrence relation in what follows

$$
f_{N}^{(k)}(z)=Z^{(k)}(z)\left(M^{-1}\right)^{\mathrm{T}} A=Z(z)\left(B^{\mathrm{T}}\right)^{k}\left(M^{-1}\right)^{\mathrm{T}} A .
$$

For the collocation points $z=z_{p q}$, the matrix relation (12) becomes

$$
f_{N}^{(k)}\left(z_{p q}\right)=Z\left(z_{p q}\right)\left(B^{\mathrm{T}}\right)^{k}\left(M^{-1}\right)^{\mathrm{T}} A ; p, q=0,1, \cdots, N .
$$

For $p=0,1, \cdots, N$ one can write the relation (13) in the following form

$$
\begin{aligned}
f_{N}^{(k)}\left(z_{0 q}\right) & =Z\left(z_{0 q}\right)\left(B^{\mathrm{T}}\right)^{k}\left(M^{-1}\right)^{\mathrm{T}} A, \\
f_{N}^{(k)}\left(z_{1 q}\right) & =Z\left(z_{1 q}\right)\left(B^{\mathrm{T}}\right)^{k}\left(M^{-1}\right)^{\mathrm{T}} A, \\
\vdots & \\
f_{N}^{(k)}\left(z_{N q}\right) & =Z\left(z_{N q}\right)\left(B^{\mathrm{T}}\right)^{k}\left(M^{-1}\right)^{\mathrm{T}} A .
\end{aligned}
$$

or briefly

$$
f_{q}^{(k)}=\left[\begin{array}{c}
f_{N}^{(k)}\left(z_{0 q}\right) \\
f_{N}^{(k)}\left(z_{1 q}\right) \\
\vdots \\
f_{N}^{(k)}\left(z_{N q}\right)
\end{array}\right] \quad q=0,1, \cdots, N,
$$

where

$$
Z_{q}=\left[\begin{array}{c}
Z\left(z_{0 q}\right) \\
Z\left(z_{1 q}\right) \\
\vdots \\
Z\left(z_{N q}\right)
\end{array}\right]=\left[\begin{array}{ccccc}
1 & z_{0 q} & z_{0 q}^{2} & \cdots & z_{0 q}^{N} \\
1 & z_{1 q} & z_{1 q}^{2} & \cdots & z_{1 q}^{N} \\
\vdots & \vdots & \vdots & \ddots & \vdots \\
1 & z_{N q} & z_{N q}^{2} & \cdots & z_{N q}^{N}
\end{array}\right]
$$

Moreover, substituting the collocation points into Equation (3), we have

$$
\sum_{k=0}^{m} P_{k}\left(z_{p q}\right) f_{N}^{(k)}\left(z_{p q}\right)=g\left(z_{p q}\right) ; p, q=0,1, \cdots, N .
$$

By means of the expressions (13) and (14), we acquire the fundamental matrix equation

$$
\sum_{k=0}^{m} \sum_{q=0}^{N} P_{k q} Z_{q}\left(B^{\mathrm{T}}\right)^{k}\left(M^{-1}\right)^{\mathrm{T}} A=\sum_{q=0}^{N} G_{q}
$$

In which

$$
P_{k q}=\left[\begin{array}{cccc}
p_{k}\left(z_{0 q}\right) & 0 & \cdots & 0 \\
0 & p_{k}\left(z_{1 q}\right) & \cdots & 0 \\
\vdots & \vdots & \ddots & \vdots \\
0 & 0 & \cdots & p_{k}\left(z_{N q}\right)
\end{array}\right]
$$

and $G_{q}=\left(g\left(z_{0 q}\right), g\left(z_{1 q}\right), \cdots, g\left(z_{N q}\right)\right)^{\mathrm{T}}$. With the aid of relation (12), we can obtain the corresponding matrix form due to the condition (4) as follows

$$
\begin{aligned}
& \sum_{k=0}^{m} \sum_{j=0}^{J}\left[a_{r k} Z\left(\xi_{j}\right)\right]\left(B^{\mathrm{T}}\right)^{k}\left(M^{-1}\right)^{\mathrm{T}} \\
& A=\left[\lambda_{r}\right] ; r=0,1, \cdots, m-1,
\end{aligned}
$$

where $Z\left(\xi_{j}\right)=\left(\begin{array}{llll}1 & \xi_{j} & \xi_{j}^{2} & \xi_{j}^{N}\end{array}\right)$.

Briefly, the system of the matrix Equation (17) can be written in the matrix form

$$
U_{r} A=\left[\lambda_{r}\right] \text { or }\left[u_{r} ; \lambda_{r}\right],
$$

where

$$
\begin{aligned}
& \sum_{k=0}^{m} \sum_{j=0}^{J}\left[a_{r k} Z\left(\xi_{j}\right)\right]\left(B^{\mathrm{T}}\right)^{k}\left(M^{-1}\right)^{\mathrm{T}} \\
& =\left[\begin{array}{llll}
u_{r 0} & u_{r 1} & \cdots & u_{r N}
\end{array}\right] ; \quad r=0,1, \cdots, m-1,
\end{aligned}
$$

We can write Equation (16) in the form

$$
W A=G \text {, }
$$

such that $W=\left[w_{s t}\right]=\sum_{k=0}^{m} \sum_{q=0}^{k} P_{k q} Z_{q}\left(B^{\mathrm{T}}\right)^{k}\left(M^{-1}\right)^{\mathrm{T}}$; $s, t=0,1, \cdots, N$, and

$$
G=\sum_{q=0}^{N} G_{q}=\left[\begin{array}{c}
g_{0} \\
g_{1} \\
\vdots \\
g_{N}
\end{array}\right],
$$

where $G_{q}$ is defined in (16). The augmented matrix of Equation (19) becomes

$$
[W ; G]=\left[w_{s t} ; g_{s}\right] ; s, t=0,1, \cdots, N .
$$

The augmented matrix of Equation (18) corresponds to $\left[U_{r} ; \lambda_{r}\right]=\left[\begin{array}{llll}u_{r 0} & u_{r 1} & \cdots & u_{r N} ; \lambda_{r}\end{array}\right]$, where $U_{r}$ is defined in (18).

Consequently, to find the unknown Hermite coefficients an, $n=0,1, \cdots, N$ related to approximate solution of the problem consisting of Equation (3) and condition (4), we replace the matrices (20) by the last $\mathrm{m}$ rows of 
the augmented matrix (19). Hence, we have a new augmented matrix $W^{*} A=G^{*}$, where

$$
\begin{aligned}
& {\left[W^{*} ; G^{*}\right]} \\
& {\left[\begin{array}{cccccc}
w_{00} & w_{01} & w_{02} & \cdots & w_{0 N} & g_{0} \\
w_{10} & w_{11} & w_{12} & \cdots & w_{1 N} & g_{1} \\
w_{20} & w_{21} & w_{22} & \cdots & w_{2 N} & g_{2} \\
\vdots & \vdots & \vdots & \ddots & \vdots & \vdots \\
w_{N-m 0} & w_{N-m 1} & w_{N-m 2} & \cdots & w_{N-m N} & g_{N-m} \\
u_{00} & u_{01} & u_{02} & \cdots & u_{0 N} & \lambda_{0} \\
u_{10} & u_{11} & u_{12} & \cdots & u_{1 N} & \lambda_{1} \\
u_{20} & u_{21} & u_{22} & \cdots & u_{2 N} & \lambda_{2} \\
\vdots & \vdots & \vdots & \ddots & \vdots & \vdots \\
u_{m-10} & u_{m-11} & u_{m-12} & \cdots & u_{m-1 N} & \lambda_{m-1}
\end{array}\right]}
\end{aligned}
$$

If $\operatorname{det}\left(W^{*}\right) \neq 0$ then we can write $A=\left(W^{*}\right)^{-1} G^{*}$. The unknown Hermite coefficients matrix $A$, is determined by solving this linear system and $a_{0}, a_{1}, \cdots, a_{N}$ are substituted in Equation (3). Thus, we obtain the Hermite polynomial solution $f_{N}(z)=\sum_{n=}^{N} a_{n} H_{n}(z)$.

\section{Comparison of Matrix Polynomial Collocation Methods}

Theorem3.1. Let $B=\left\{\beta_{i}\right\}_{i=0}^{k}$ be a base for vector space $S$, then every member $S \in S$ has a unique representation in the form of linear combination of these vectors.

\section{Proof. [13].}

Based on the above theorem, if the bases of approximate space in collocation methods are chosen from complex polynomials up to degree $N$, using different bases or choosing of different complex polynomial classes as the base has no effect on the approximate solution, theoreticcally. This means that if $\left\{\varphi_{n}(z)\right\}_{n=0}^{N}$ and $\left\{\varphi_{n}^{\prime}(z)\right\}_{n=0}^{N}$ be two different bases according to the uniqueness representation, then approximate solution of (1) can be written

$$
f_{N}(z)=\sum_{n=0}^{N} a_{n} \varphi_{n}(z)=\sum_{n=0}^{N} a_{n}^{\prime} \varphi_{n}^{\prime}(z) .
$$

For this reason when we use different polynomials (such as Taylor, Bessel, Hermite, etc.) in polynomial Collocation methods one expects the equal results obtained.

In the numerical implementation, to determine coefficients an, $n=0,1, \cdots, N$ in (5), we should solve a system of equations in the form of $W F=G$ and properties of matrix $W$ is directly depended on choosing the base. So different bases result different matrix $W$ with different properties. Some of these properties such as condition number has the direct influence on solution's accuracy. In addition CPU time for solving these systems differs for different bases. Hence, different polynomial bases can cause solutions with different accuracy.

Our experiences show that when we use different polynomial classes in matrix polynomial collocation methods, there is negligible difference among approximated solutions. In Section 4, we compare this matter for several examples by using Taylor, Bessel and Hermite polynomials.

\section{Numerical Examples}

Several numerical examples are studied in this section to illustrate the accuracy and efficiently properties of Taylor, Bessel and Hermite collocation method. In this paper, collocation points in the rectangular domain (3) are defined by $z_{p q}=x_{p}+i y_{q}$, such that

$$
\begin{aligned}
& x_{p}=a+\frac{b-a}{N} p, y_{q}=c+\frac{d-c}{N} q, a \leq x_{p} \leq b, \\
& c \leq y_{q} \leq d ; p, q=0,1, \cdots, N
\end{aligned}
$$

and in the elliptic domain (4) are defined by

$$
\begin{aligned}
& z_{p q}=x_{p q}+i y_{p q}=\left(x_{p q}, y_{p q}\right) \in D, \\
& x_{p q}=\frac{a}{N} p \cos \frac{\theta}{N} q, \\
& y_{p q}=\frac{b}{N} p \sin \frac{\theta}{N} q,
\end{aligned}
$$

such that $p, q=0,1, \cdots, N ; 0 \leq q \leq 2 \pi$, $z_{0 q}=z_{00}=(0,0)$.

Examples show that the difference among collocation methods based on these polynomials is negligible. All of them are performed on a computer using programs written in MATLAB 2011a. In this regard, we have reported in the Tables the value of absolute error function $e_{N}(z)=\left|f(N)-f_{N}(z)\right|$ at the selected points of the domain.

\subsection{Example 1}

As the first example, [10], we consider the linear second order complex differential equation

$$
\begin{aligned}
& \left(1-z^{2}\right) f^{\prime \prime}(z)-2 z f^{\prime}(z)+6 f(z) \\
& =\left(z^{2}+5\right) \cos (z)+2 z \sin (z),
\end{aligned}
$$

with $f(0)=\frac{1}{2}, f^{\prime}(0)=0$ and exact solution

$f(z)=\frac{3}{2} z^{2}-\frac{1}{2}+\cos (z)$ on elliptic domain with

$a=1, b=\frac{1}{2}$ and $\theta=\pi$. Absolute errors are listed in

Table 1. 

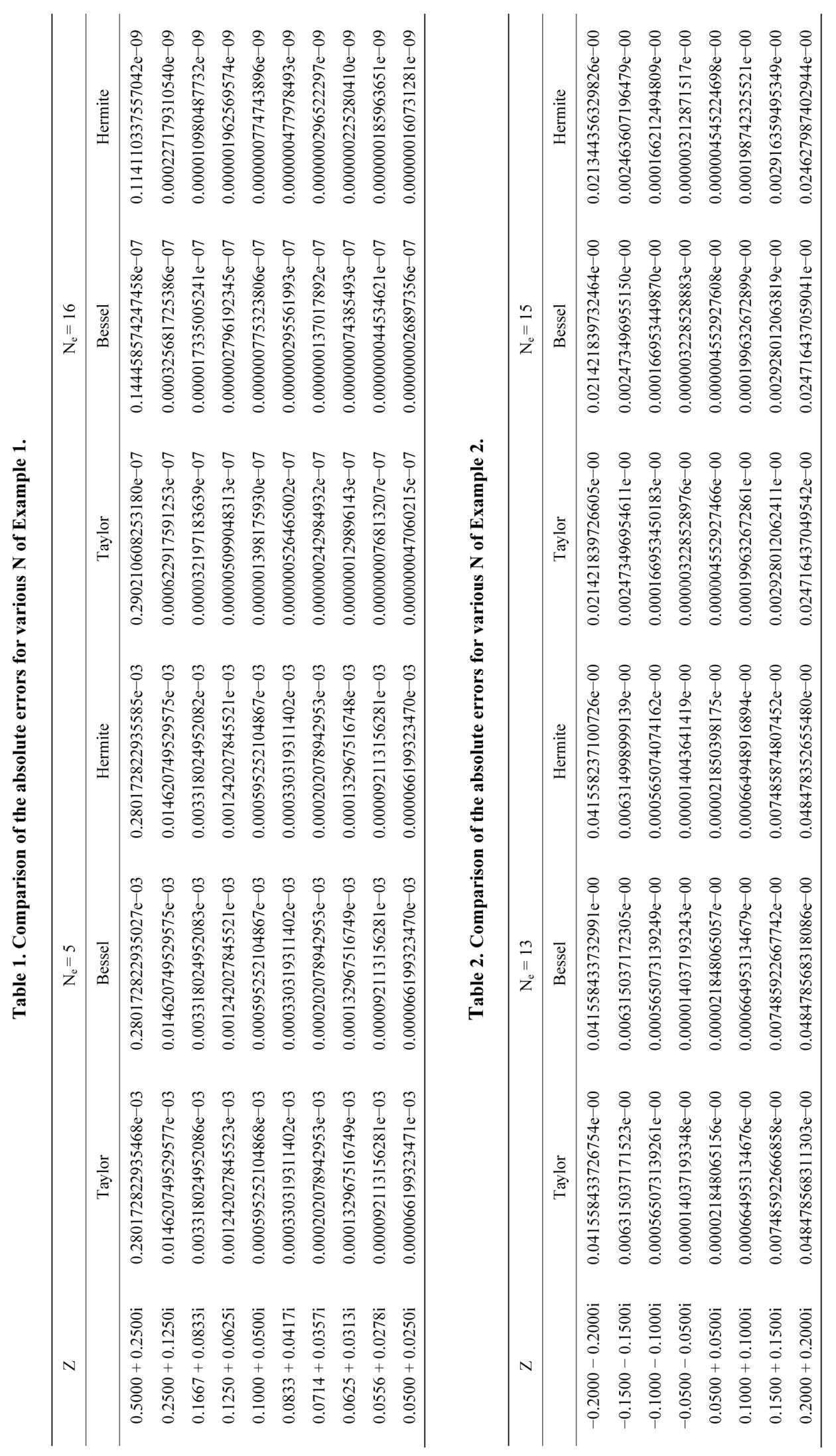

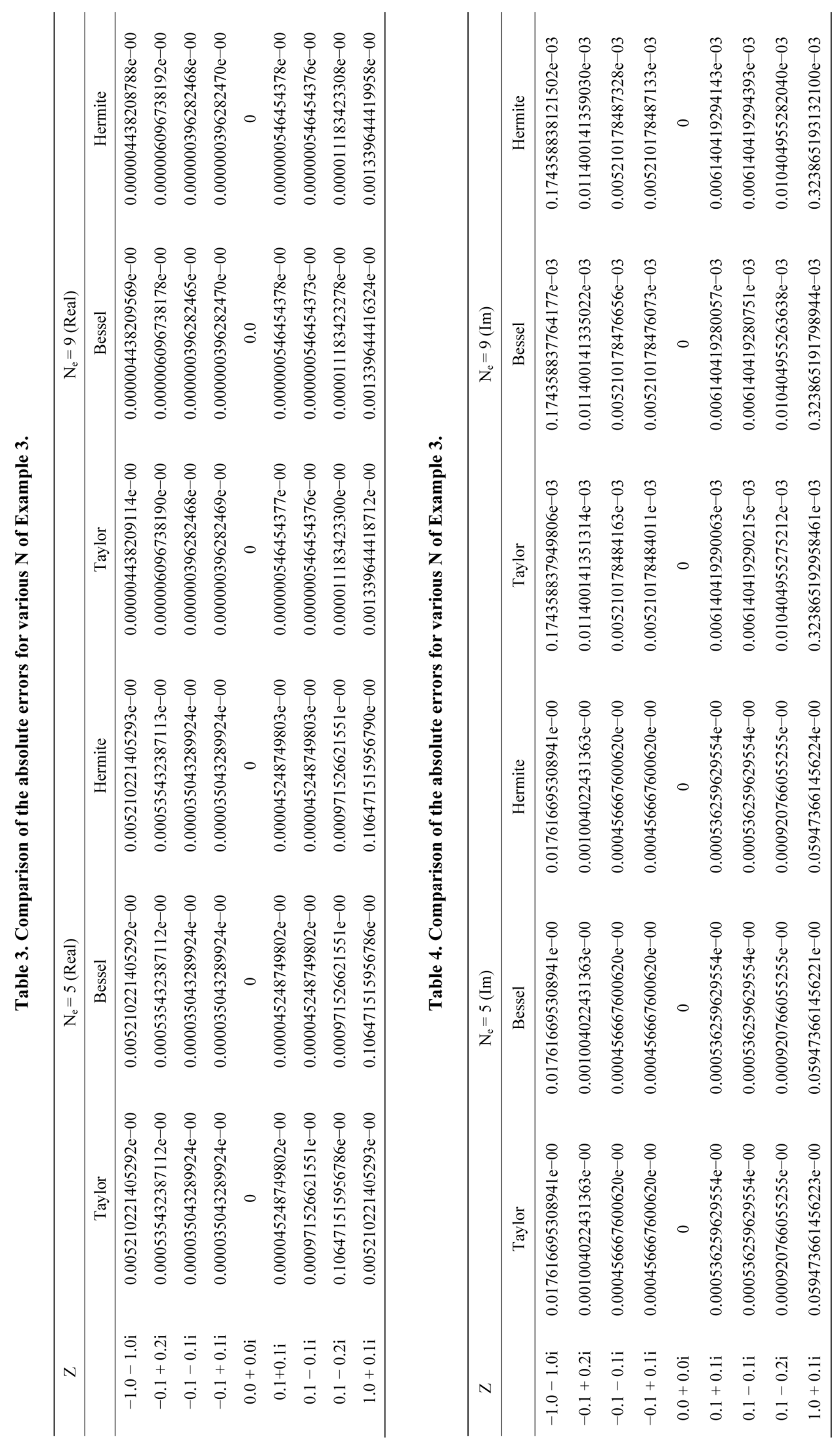


\subsection{Example 2}

In this example, [10], we consider the third order linear complex differential equation

$$
\begin{aligned}
& f^{\prime \prime \prime}(z)-f^{\prime}(z) \\
& +\left(-8 \mathrm{e}^{6 z}-12 \mathrm{e}^{5 z}-30 \mathrm{e}^{4 z}-19 \mathrm{e}^{3 z}-9 \mathrm{e}^{2 z}\right) f(z)=0,
\end{aligned}
$$

with the condition $f(0)=\mathrm{e}^{2}, f^{\prime}(0)=3 \mathrm{e}^{2}$, $f^{\prime \prime}(0)=14 \mathrm{e}^{2}$ and exact solution $f(z)=\mathrm{e}^{2 z}+\mathrm{e}^{z}$ in elliptic domain with $a=1, b=\frac{1}{2}$ and $\theta=\pi$. Absolute errors of the obtain solutions are given in Table 2 .

\subsection{Example 3}

The last example, [11] is the second order complex differential equation

$$
f^{\prime \prime}(z)+z f(z)=\mathrm{e}^{z}+z \mathrm{e}^{z},
$$

with the initial conditions $f(0)=1, f^{\prime}(0)=1$. The exact solution is $f(z)=\mathrm{e}^{z}$ on rectangular domain with $a=-1, b=1, c=-1, d=1$. Absolute errors are listed in Tables 3 and 4 .

\section{Conclusion}

In this article, approximate solutions which can be obtained by different polynomial collocation methods have been compared. Our experiments show that using different polynomials cannot significantly affect the numerical solutions and the results are similar to each other.

\section{REFERENCES}

[1] G. Barsegian, "Gamma-Lines: On the Geometry of Real and Complex Functions," Taylor and Frencis, London, 2002.

[2] G. Barsegian and D. T. Le, "On a Topological Description of Solutions of Complex Differential Equations," Complex Variables, Vol. 50, No. 7-11, 2005, pp. 307-318. http://dx.doi.org/10.1080/02781070500086743

[3] K. Ishisaki and K. Tohge, "On the Complex Oscillation of Some Linear Differential Equations," Journal of Mathematical Analysis and Applications, Vol. 206, No. 2,
1997 , pp. 503-517. http://dx.doi.org/10.1006/jmaa.1997.5247

[4] J. Heittokangas, R. Korhonen and J. Rättyä, “Growth Estimates for Solutions of Linear Complex Differential Equations," Annales Academiae Scientiarum Fennicae Mathematica, Vol. 29, No. 1, 2004, pp. 233-246.

[5] V. A. Prokhorov, "On Best Rational Approximation of Analytic Functions," Journal of Approximation Theory, Vol. 133, No. 2, 2005, pp. 284-296. http://dx.doi.org/10.1016/j.jat.2004.12.007

[6] V. Andrievskii, "Polynomial Approximation of Analytic Functions on Finite Number of Continua in the Complex Plane," Journal of Approximation Theory, Vol. 133, No. 2, 2005, pp. 238-244. http://dx.doi.org/10.1016/j.jat.2004.12.016

[7] M. Sezer and M. Güsu, "Approximate Solution of Complex Differential Equations for a Rectangular Domain with Taylor Collocation Method," Applied Mathematics and Computation, Vol. 177, No. 2, 2006, pp. 844-851. http://dx.doi.org/10.1016/j.amc.2005.11.035

[8] M. Güsu and M. Sezer, "Approximate Solution to Linear Complex Differential Equations by a New Approximate Approach," Applied Mathematics and Computation, Vol. 185, No. 1, 2007, pp. 636-645. http://dx.doi.org/10.1016/j.amc.2006.07.050

[9] M. Sezer and S. Yalcinbas, "A Collocation Method to Solve Higher Order Linear Complex Differential Equations in Rectangular Domains," Numerical Methods for Partial Differential Equations, Vol. 26, No. 3, 2010, pp. 596-611.

[10] M. Sezer, B. Tanay and M. Güsu, "Numerical Solution of a Class of Complex Differential Equations by the Taylor Collocation Method in Elliptic Domains," Numerical Methods for Partial Differential Equations, Vol. 26, No. 5, 2010, pp. 1191-1205.

[11] S. Yüzbasi, N. Sahin and M. Sezer, "A Collocation Approach for Solving Linear Complex Differential Equations in Rectangular Domain," Mathematical Methods in the Applied Sciences, Vol. 35, No. 10, 2012, pp. 11261139. http://dx.doi.org/10.1002/mma. 1590

[12] M. Güsu, H. Yalman, Y. Ozturk and M. Sezer, “A New Hermite Collocation Method for Solving Differential Difference Equations," Applications and Applied Mathematics, Vol. 6, No. 1, 2011, pp. 116-129.

[13] K. Haffman and R. Kunze, "Linear Algebra," 2nd Edition, Prentice-Hall, Upper Saddle River, 1971. 